\title{
Electronic Interface for Lidar System and Smart Cities Applications
}

\author{
L. Pantoli, Member, IEEE, G. Barile, Student Member, IEEE, A. Leoni, Student Member, IEEE, \\ M. Muttillo, Student Member, IEEE, and V. Stornelli, Senior Member, IEEE
}

\begin{abstract}
This work deals with the design of a new readout electronics for silicon photomultipliers sensors. The so-called SiPMs sensors are an emerging technology currently diffusing in many applications and, among them, in the definition of a new generation of LIDAR systems. The latter, nowadays have a primary role in the evolution process that is involving Smart Cities, being an enabling technology in different fields. The solution here proposed is realized at electronic level with a $150 \mathrm{~nm}$ technology process from LFoundry and results provide a feasible demonstration of the capability of the proposed design approach to be employed in practical applications.
\end{abstract}

Index Terms-Sensor interface, Lidar, sensors system, SiPM, Smart City.

\section{INTRODUCTION}

$\mathrm{L}$ IDAR (Light Detection and Ranging) is a new enabling technology that is rapidly spreading and establishing in many applications. It allows to have high accuracy and resolution at long distance, also in complex environmental conditions, as with bright light and reflective surfaces or poor visibility [1-6]. The main fields of application are mobile range findings, automotive with advanced driver assistance systems, 3D mapping, motion detection and recognition.

Initially, LIDAR systems have been realized in different technologies and by employing different types of sensors such as PIN diodes, photodiodes or vacuum-tube photomultipliers [7-8]. But nowadays, current applications are demanding even more performance and so among the cited solutions the ones that can be still used with proficiency are diminishing; while further solutions have been developed. Among them, a primary role is now occupied by Silicon Photomultipliers (SiPMs) [912]; SiPM is a new leading technology for light detection that is capable to furnish high accuracy and resolution together with low power consumption. It is particularly suitable for portable and mobile applications.

Manuscript received January 14, 2019; revised March 5, 2019. Date of publication April 11, 2019. Date of current version June 3, 2019.

Authors are with the Department of Industrial and Information Engineering and Economics of University of L'Aquila, L'Aquila, 67100 Italy.

E-mails: \{leonardo.pantoli, vincenzo.stornelli\}@ univaq.it, \{alfiero.leoni, gianluca.barile, mirco.muttillo\}@graduate.univaq.it.

Digital Object Identifier (DOI): 10.24138/jcomss.v15i2.692
In addition, SiPMs have very high internal gain and this characteristic allows to avoid the use of additional amplifiers that may limit the signal-to-noise ratio providing an additional noise contribution. On the contrary, between the shortcomings of these devices, it is important to consider the high parasitics of the SiPMs sensors that require strict performance from the electronics side in order to properly work [13-16].

Also this kind of photomultipliers are currently diffusing in several fields, from astrophysics to imagine scanning, from medical application to automotive embarked in LIDAR systems.

Actually, there is a huge demand of LIDAR applications; not only in automotive but also in the fields of robotics, laser scanning and drones. The development of Smart Cities itself is one of the main factors that powers this technology and the definition of novel applications. It affects almost all systems and technologies, as well as the same infrastructures, and so Smart Cities represent a leading road towards innovations. Considering, for instance, the definition of the new wireless data networks for connected-systems in urban environment, LIDAR systems are the primary solution to obtain an accurate 3D urban analysis for propagation modelling and design [1720]. Similar advantages outcome from the data needed to determine the challenges that are associated with climate change resilience in urban metropolis [21]. Also the intelligent transportation systems under definition in modern developed cities are strongly based on LIDAR systems [22-24]. In general, the wish of increased service efficiencies intrinsic in Smart Cities can be realized through advanced technologies and systems, functional to the new needs of the urban society.

In this paper the attention is focused on the definition of a novel sensor interface for LIDAR systems that uses SiPMs as sensing elements. As already mentioned, this kind of system has powerful performance in term of both detection capability, mobility and power consumption, but from an electronic point of view it is difficult to manage and emphasize. It is due to the electrical characteristics of the light sensor. The solution here proposed allows a fast detection of any light phenomenon with a simple architecture able to transform the incoming light event revealed with a current pulse in a corresponding voltage signal that can be easily managed and analyzed for detection. 
The designed circuit is realized with the silicon $150 \mathrm{~nm}$ technology process provided by LFoundry demonstrating the feasibility of the proposed solution to be adopted in practical applications and integrated systems.

The paper is organized as followings. Section II provides an overview of LIDAR systems based on SiPMs sensors; Section III deals on the design of the proposed sensor interface, while Section IV shows the obtained results and discuss the achieved performance. Finally, conclusions are drawn.

\section{SIPM-BASED LIDAR OVERVIEW}

The Silicon Photomultiplier is a miniaturized solid-state sensor for light detection, with very high sensitivity and detection capability of energy levels typical of a single photon. SiPM is realized with a series of avalanche photodiodes (usually named SPAD: Single Photon Avalanche Photodiode) combined with quench resistors (Fig. 1). This kind of sensor provides high gain with good efficiency and has a very large bandwidth $[25,26]$ that allows to achieve an agile response when an event occurs. In addition, SiPMs sensors can be arranged also in matrix configuration in order to increase the sensitive area and the energy level detection capability.

There is an even more number of fields of interest that may benefit of the SiPM characteristics. In particular, LIDAR applications, by using SiPMs, are able to realize high-resolution object identification at long range, also as portable devices. They operate near infrared wavelength and are commonly applied to driver assistance systems or autonomous guide vehicles, 3D depth maps, mobile ranging. Typical characteristics of a LIDAR system are summarized in Table 1, while an example of application is shown in Fig. 2 with a direct time-of-flight ranging architecture. The transmitter embeds a synchronous laser diode and some lens and filters able to collimate the light beam on the target. The detector catches the reflected ray of light and with lens conveys the incoming radiation to the sensitive surface of the SiPM.

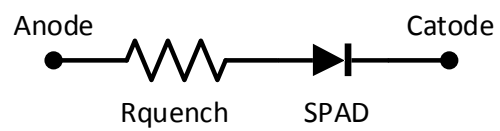

(a)

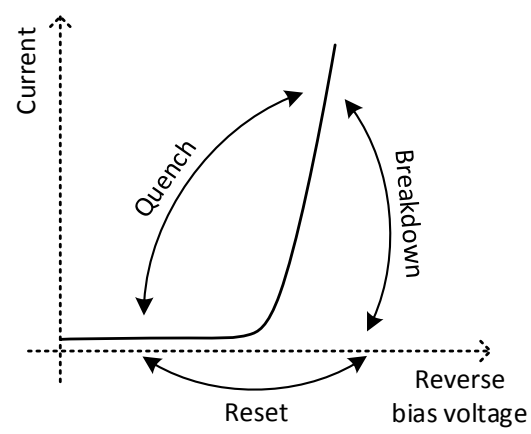

(b)

Fig. 1. SiPM characteristics: (a) electrical scheme; (b) Output current versus bias voltage.
TABLE I

LIDAR DESIGN WITH SIPM: TYPICAL CHARACTERISTICS

\begin{tabular}{cc}
\hline \hline Characteristics & Typical choices \\
\hline Beam Steering & $1 \mathrm{D}$ or $2 \mathrm{D}$ \\
Ranging solutions & $40^{\circ} \times 30^{\circ}$ Long range \\
& $120^{\circ} \times 30^{\circ}$ Short range \\
Sensor Specification & $1 \times 16 \mathrm{SiPM}$ \\
Resolution & AoVx $=0.1^{\circ}$ \\
& AoVy $=1.9^{\circ}$ \\
Optical bandpass filter & 10 to $50 \mathrm{~nm}$ \\
Laser diode peak power & $1000 \mathrm{~s} \mathrm{~W}$ \\
Laser wavelength & $905 \mathrm{~nm}$ or $940 \mathrm{~nm}$ \\
Laser pulse repetition rate & $100 \mathrm{~s} \mathrm{KHz}$ \\
Laser pulse width & Less than $10 \mathrm{~ns}$ \\
Bias voltage & $<50 \mathrm{~V}$ \\
\hline \hline
\end{tabular}

A subsequent electronic readout system is then necessary to properly manage the light event and convert it to an electrical signal useful to detect and decode the received information. The electronic section is usually organized with an input amplifier usually followed by a buffer and a detector realized with a comparator. In addition, a cross logic section responsible of data management and synchronization of the overall system is also necessary.

Among them, the more critical part to design in order to take advantage of the sensing capability of the SiPM is the initial amplification section. It realizes also the signal transduction since the output signal of the SiPM is a very low current signal and the same SiPM has very large parasitics that can be usually modeled with an output capacitance. This characteristic, from an electrical point of view, is far from an ideal condition, since the following amplification stage must be capable to drive a very large input load; at the same time, it has to provide a sufficient gain in a large bandwidth and without introducing a significant noise contribution to properly discriminate the low input signal from the electronic noise floor. The aim to provide a feasible solution to this issue is the main goal of this contribution and it will be discussed in the next section.

\section{PROPOSED SENSOR INTERFACE}

The readout electronic for SiPMs is a crucial task for LIDAR systems since the optical detection must be properly transduced to an electrical signal in order to properly caught the information. In order to have a good resolution and properly detect objects at high distance fast electronics circuits are mandatory. They mast be able to manage very weak signals and also to operate up to high frequencies since the received pulses have critical shapes with very high rise-time and short duration, as shown in Table 1.

Typically, in the literature, the solutions usually realized can be set in the frame of voltage-mode design [27-30] and this because a voltage-approach is well consolidated and commonly adopted in the design of sensor interfaces. Without any doubts, voltage-design is able to provide robust solutions, with good noise performance and gain, but at the same time it suffers of some drawbacks. In particular, suitable strategies are required 


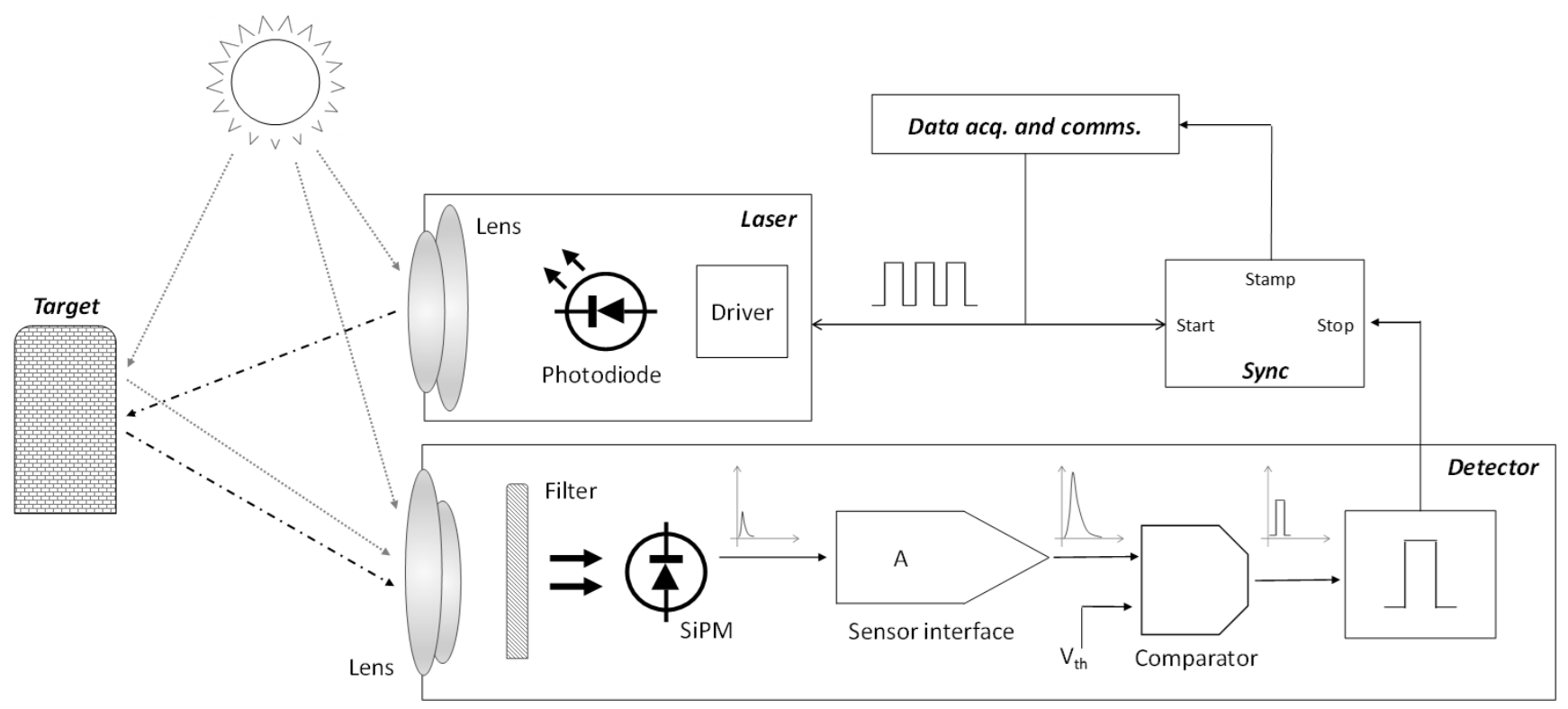

Fig. 2. Example of application: direct time-of-flight ranging LIDAR system.

to accomplish the capability to properly drive a very high input reactive load, mainly capacitive in case of SiPMs. In addition, an output buffer is also necessary to properly decouple the circuit characteristics from the output load impedance and this leads to a further increase of the circuit dimensions and power consumption. On the opposite, current-mode design has the advantage to provide a faster response time with respect to a voltage-approach [31] and also the driving capability should be better when considering small signals together with a high input impedance. As a disadvantage, the main criticism is relative to the higher noise contribution introduced with a current-mode circuit [30].

In this work, the solution that has been investigated is a mixed-mode design that try to inherit the advantages of both voltage-mode and current-mode design. Preliminary solutions have been already proposed by some of the same Authors in [32-33]. Here the analysis and design have been carried out by using an integrated technology process, the CMOS $150 \mathrm{~nm}$ technology process from LFoundry, showing at the same time the feasibility of the proposed solution to be realized at

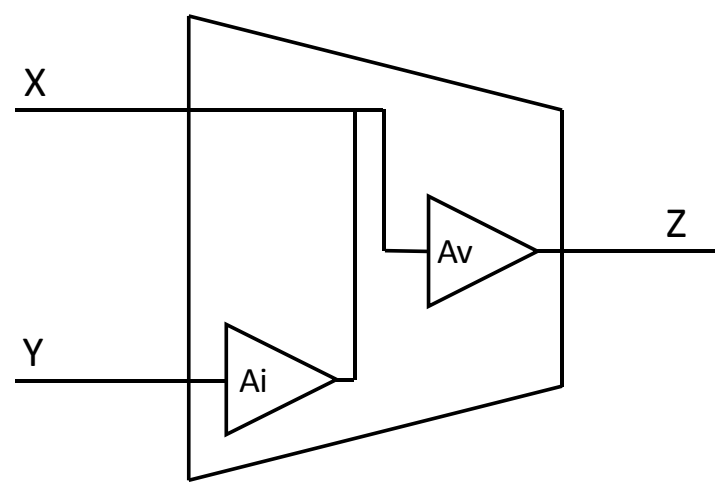

Fig. 3. VCII architecture: internal structure. integrated level, that is the main goal for practical applications. The solution here adopted is based on the use of a secondgeneration current conveyor (VCII) [34-35], obtained for duality from the second-generation current conveyor (CCII) [36-37]. The VCII realizes internally a current buffer followed by a voltage buffer (Fig. 3), so obtaining a transimpedance amplification between input and output and low input and output impedances. As shown, this building block has 3 external terminals, $X$ and $Y$ are the input ports and $Z$ is the output port. $X$ is a high impedance termination, while $Y$ and $Z$ have ideally zero impedance. The voltage gain between $X$ and $Z$ is usually called $\alpha$, while the current gain between $Y$ and $X$ terminals can be defined as $\beta$; both of them are ideally equal to unit. The constitutive equations of the VCII are the ones in (1).

$$
\begin{aligned}
& V_{Z}=\alpha \cdot V_{X} \\
& I_{X}=\beta \cdot I_{Y}
\end{aligned}
$$

Combining (1a) and (1b) and considering a load resistor $R$ in series connection on the $X$ terminal as shown in Fig. 4, the complete transimpedance transfer function can be obtained as follows:

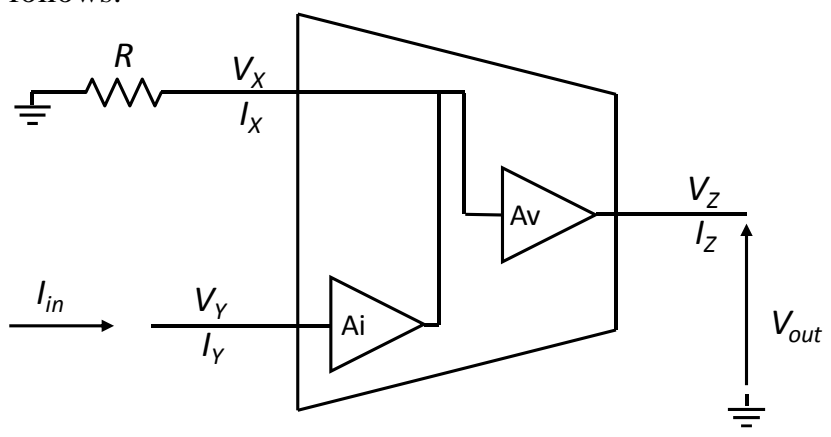

Fig. 4. VCII as transimpedance amplifier. 


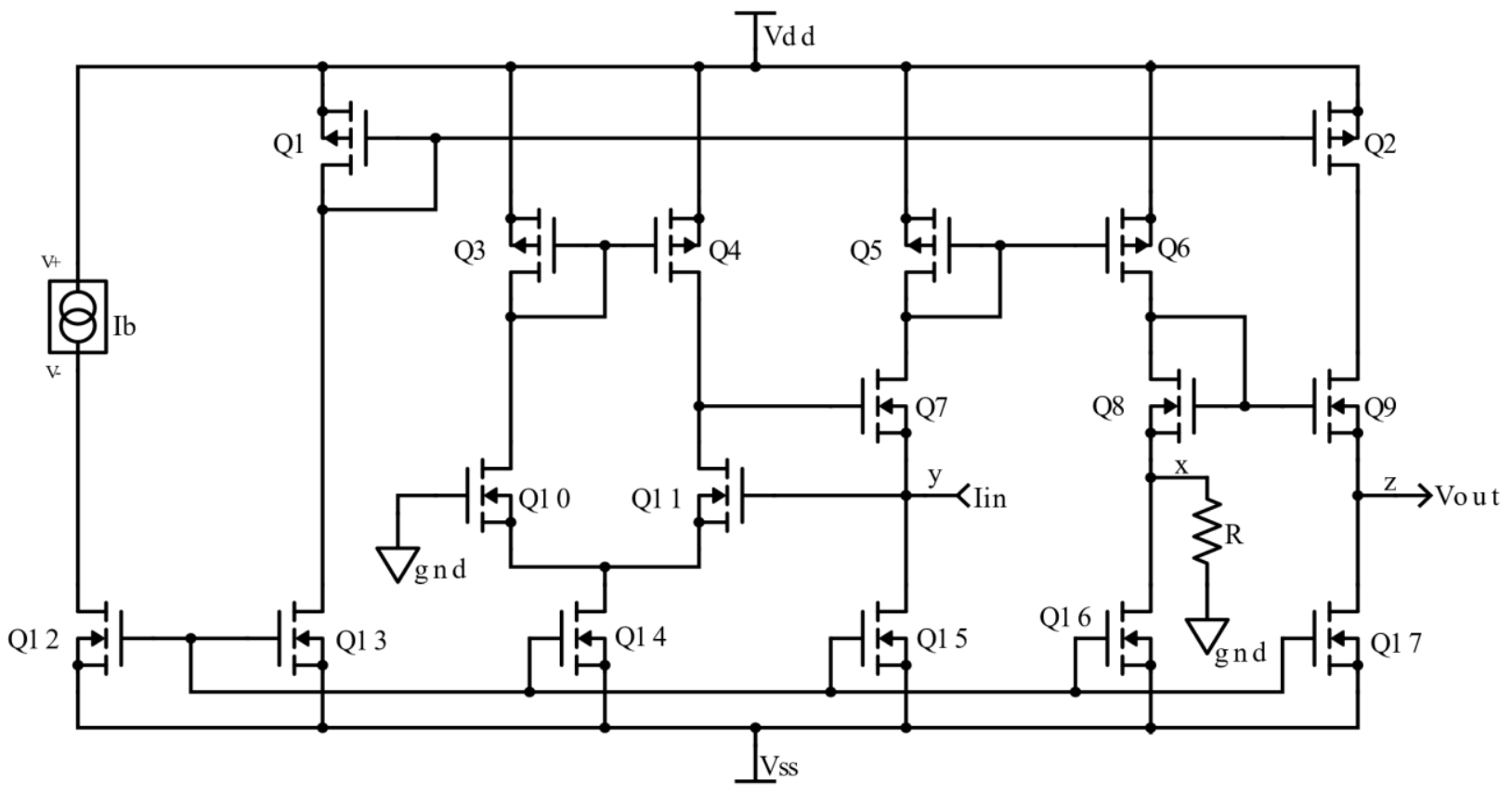

Fig. 5. Complete schematic of the proposed SiPM interface.

TABLE II

TRANSISTORS SIZES

\begin{tabular}{cc}
\hline \hline Devices & Dimensions \\
$W, L$
\end{tabular}

$$
\begin{gathered}
V_{\text {out }}=V_{Z}=\alpha \cdot V_{X}=\alpha \cdot R \cdot I_{X}=\alpha \cdot R \cdot \beta \cdot I_{Y} \\
=\alpha \cdot R \cdot \beta \cdot I_{\text {in }}
\end{gathered}
$$$$
\frac{V_{\text {out }}}{I_{\text {in }}}=\alpha \cdot \beta \cdot R
$$

Equation (3) clearly shows as the transimpedance gain depends only on the gain factors $\alpha$ and $\beta$, usually close to one on a large bandwidth, and on the resistance value $R$ in series connection on the $X$ terminal. So, the gain is independent from input and output external loads. In addition, it is important to notice that the VCII has the further characteristic of having the gain value independent from the useful bandwidth [34] and also this peculiarity makes it particularly suitable for the considered application.

At schematic level, the VCII has been designed with LFoundry technology with $150 \mathrm{~nm}$ gate length devices and the complete schematic of the proposed SiPM interface is reported in Fig. 5, while in Table II the transistors sizes are reported for completeness. Finally, Table III summarizes the main performance and bias characteristics of the proposed sensor interface.

TABLE III

CIRCUIT MAIN CHARACTERISTICS

\begin{tabular}{cc}
\hline \hline Parameter & Value \\
\hline$I_{b}$ & $30 \mu \mathrm{A}$ \\
Static power consumption & $309 \mu \mathrm{W}$ \\
$Z_{X}(@ \mathbf{1 k H z})$ & $230 \mathrm{k} \Omega$ \\
$Z_{Y}(@ \mathbf{1 k H z})$ & $256 \Omega$ \\
$Z_{Z}(@ \mathbf{1 k H z})$ & $890 \Omega$ \\
$\boldsymbol{\alpha}(@ \mathbf{k H z})$ & $-313 \mathrm{mdB}$ \\
$\boldsymbol{\beta}(@ \mathbf{1 k H z})$ & $-118 \mathrm{mdB}$ \\
\hline \hline
\end{tabular}




\section{Simulation Results}

In this section, simulation results relative to the proposed VCII and the complete SiPM interface are shown. The design has been carried out by using LTSpice simulation software provided by Analog Devices, Inc. Next Fig. 6 -8 refer to the VCII characteristics, while from Fig. 9 to Fig. 13 system performance are reported. More in details, Fig. 6 depicts the $\alpha$ parameter of the VCII, that is the gain of the voltage buffer between the $X$ and $Z$ nodes. As shown, the available bandwidth is very large, greater than one gigahertz. Also the $\beta$ characteristics are valuable, as reported in Fig. 7 and Fig. 8. The first one shows the current buffer transfer function between the $Y$ and $\mathrm{X}$ nodes and also in this case the bandwidth is very large,

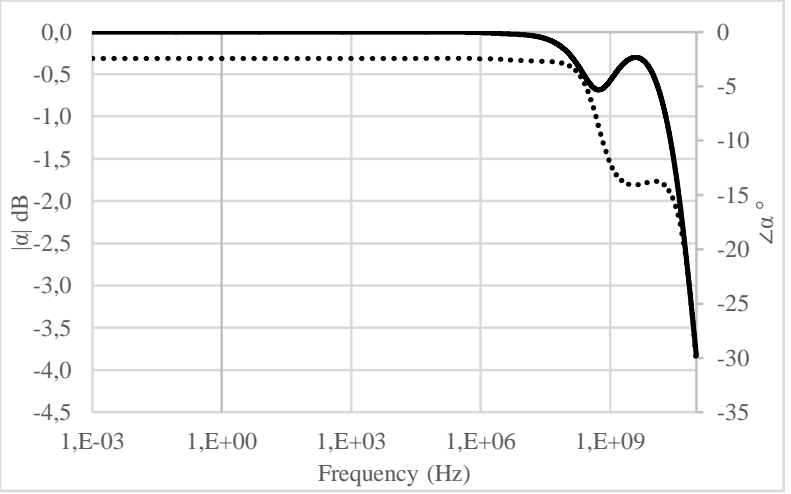

Fig. 6. $\alpha$ parameter (Magnitude in solid line; phase in dotted line).

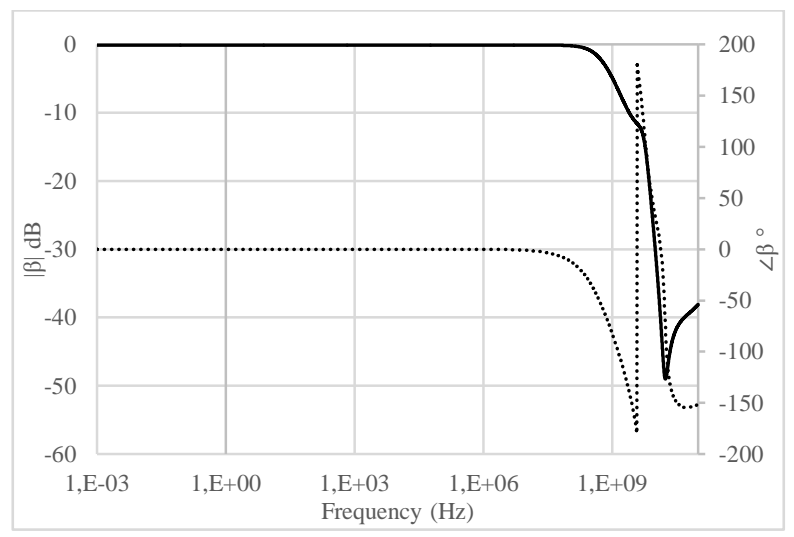

Fig. 7. $\beta$ parameter (Magnitude in solid line; phase in dotted line).

while the second picture reports the $\beta$ characteristics versus the input load, usually a very large capacitive impedance. This is a crucial point of the design for practical applications and results show the robustness of the proposed solution. Figure 9 illustrates the overall transimpedance transfer function of the complete interface. It shows the capability to set the desired gain by varying only the resistance value $\mathrm{R}$ of the resistor connected to the $\mathrm{X}$ terminal in the complete schematic of Fig. 4. As can be easily verified the gain variation slightly affects the useful bandwidth of the interface, and so the response time of the circuit. For completeness, also input and output impedances are reported in Fig. 10. The low impedance achieved in particular at the input terminal is coherent with the theoretical analysis of the VCII circuit and it confirms the driving capability of the readout electronics.

Further interesting aspects to analyze are the noise characteristics and the analysis of the impulse response. Noise performances are reported in Fig. 11, again for different value of the resistor $\mathrm{R}$ connected to the $X$ terminal, that means for different gain levels of the transimpedance amplifier. The noise level introduced by the proposed interface is very marginal and almost constant with respect to gain variations.

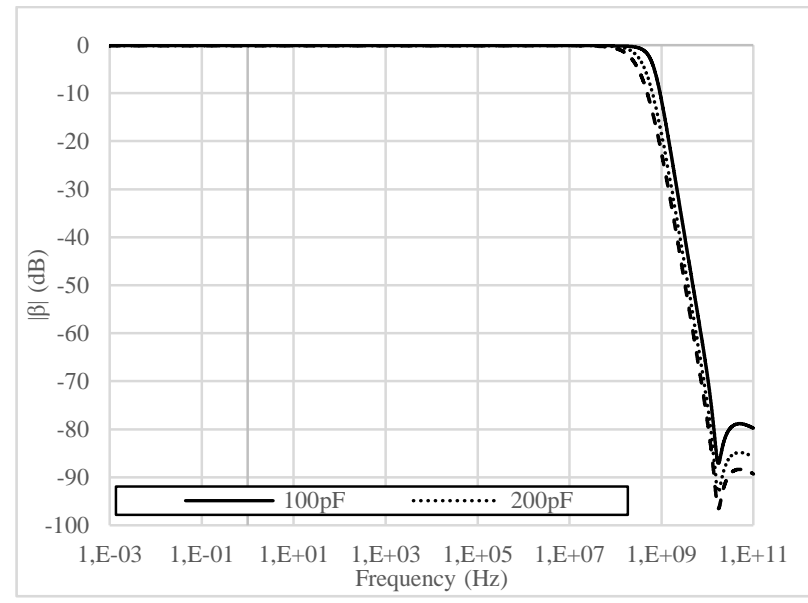

Fig. $8 . \beta$ vs input capacitive load.

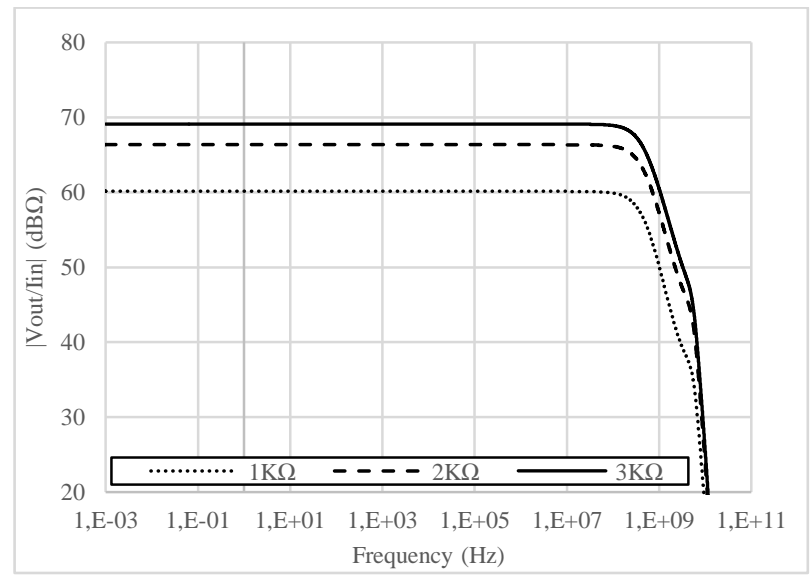

Fig. 9. Variable gain: gain versus resistance value R.

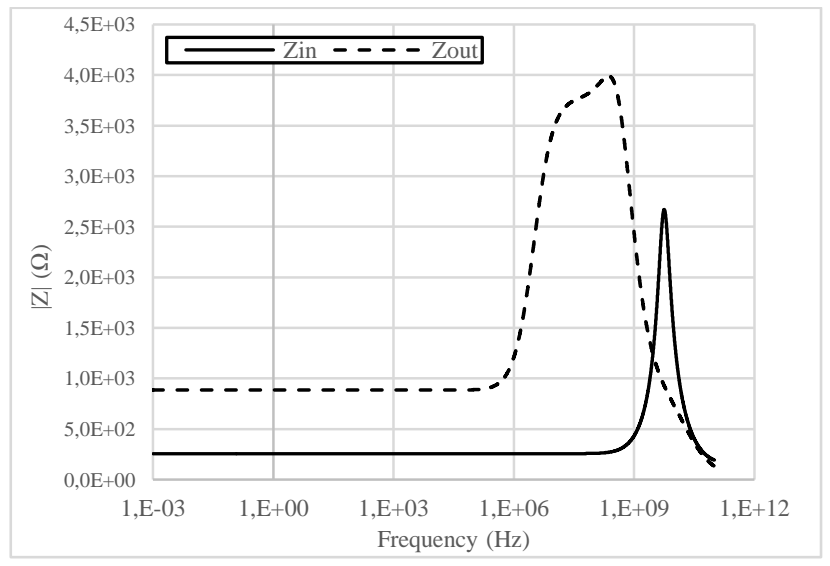

Fig. 10. Input and output impedances of the sensor interface. 


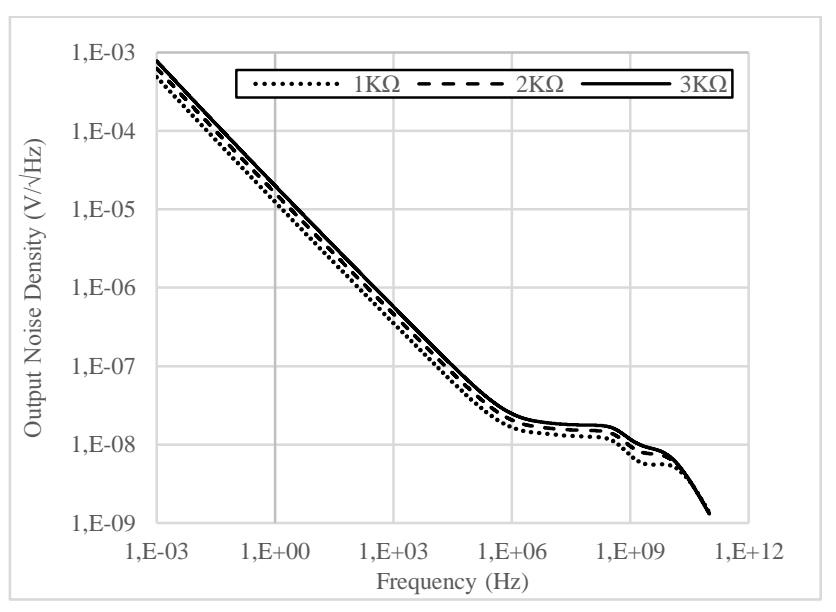

Fig. 11. Noise performance for different gain levels.
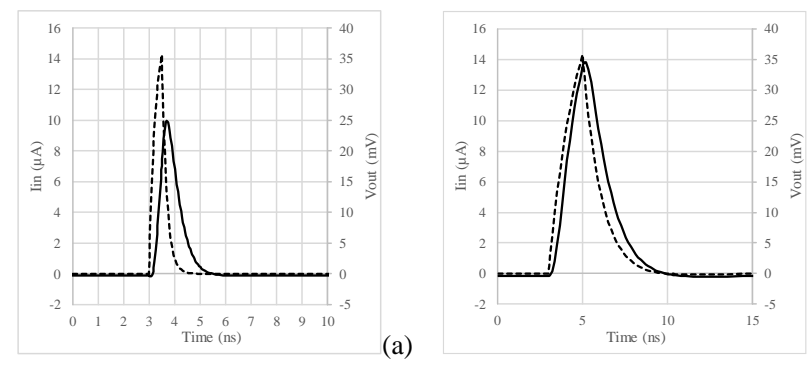

(a)
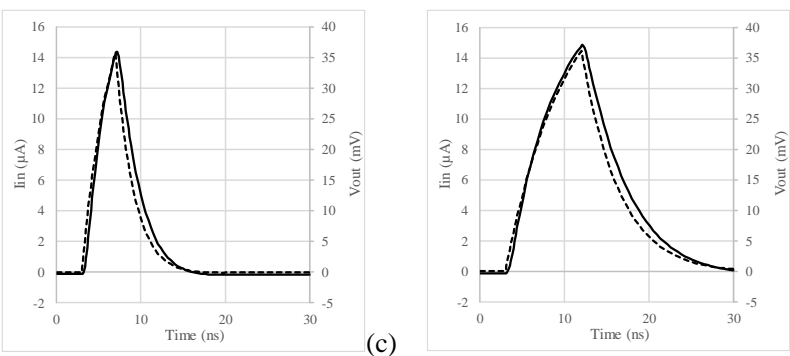

(b)

Fig. 12. Output signal (solid line) for different duration of the incoming current pulse (dotted line): (a) $1 \mathrm{~ns}$; (b) $7 \mathrm{~ns}$; (c) $15 \mathrm{~ns}$; (d) $30 \mathrm{~ns}$.

Finally, time domain analyses have been performed in order to analyze the response time of the circuit considering typical pulses as used in LIDAR systems [25, 26]. In Fig. 12 subfigures, incoming current pulses of different duration are considered and in each case the output voltage signal is very similar to the input one, so demonstrating a good detection capability with also a neglectable delay; this is another important parameter in practical applications and mainly in LIDAR systems. The pulse duration can be reduced up to $1 \mathrm{~ns}$ still preserving good performance and in general it can be affirmed that the lower boundary is mainly due to technology limitation rather than a physical limit of the proposed interface. Figure 13 demonstrates also the capability of the system in the case of multi-phonon detection. An incoming pulse train defined with a single pulse of $10 \mathrm{~ns}$ length and a repetition time of $20 \mathrm{~ns}$ is considered and results clearly show the tracking and detection capability of the designed interface.

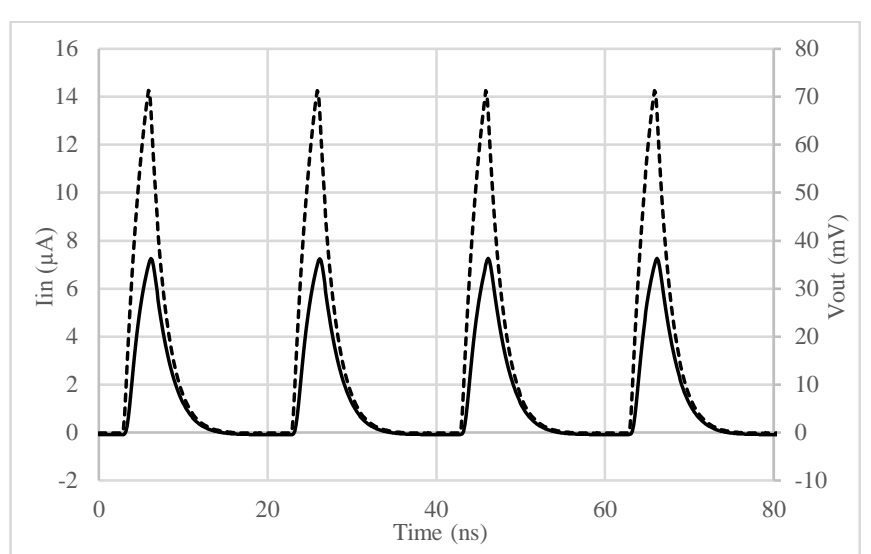

Fig. 13. Output signal (solid line) in case of an incoming pulse train (dotted line).

\section{CONCLUSION}

In the perspective of innovations relative to Smart Cities, with no doubt LIDAR systems have a primary role enabling new technologies in different fields of applications. The proposed work shows a new feasible solution for the design of the readout electronics of LIDAR systems allowing to obtain fast response times and high sensitivity. These characteristics, combined with the use of the new SiPMs sensors, enhance the performance of many systems, from intelligent drive to $3 \mathrm{D}$ mapping. At electrical level we propose an integrated solution realized with the $150 \mathrm{~nm}$ technology process from LFoundry that realize a transimpedance amplifier with very high driving capability, a very large bandwidth and a variable gain. The solution is conceived with a mixed voltage/current-mode approach that is able to provide a very fast response time that is mandatory for the new generation of LIDAR systems.

\section{REFERENCES}

[1] J. Liu, Q. Sun, Z. Fan and Y. Jia, "TOF Lidar Development in Autonomous Vehicle," 2018 IEEE 3rd Optoelectronics Global Conference (OGC), Shenzhen, 2018, pp. 185-190, J. Liu, Q. Sun, Z. Fan and Y. Jia, "TOF Lidar Development in Autonomous Vehicle," 2018 IEEE 3rd Optoelectronics Global Conference (OGC), Shenzhen, 2018, pp. 185-190, DOI: 10.1109/OGC.2018.8529992.

[2] J. R. Santillan, M. Makinano-Santillan and L. C. Cutamora, "Integrating LiDAR and flood simulation models in determining exposure and vulnerability of buildings to extreme rainfall-induced flood hazards," 2016 IEEE International Geoscience and Remote Sensing Symposium (IGARSS), Beijing, 2016, pp. 7585-7588, DOI: 10.1109/IGARSS.2016.7730978.

[3] S. Gargoum and K. El-Basyouny, "Automated extraction of road features using LiDAR data: A review of LiDAR applications in transportation," 2017 4th International Conference on Transportation Information and Safety (ICTIS), Banff, AB, 2017, pp. 563-574, DOI: 10.1109/ICTIS.2017.8047822.

[4] Y. Zhang, S. Gu, J. Yang, M. Jose Alvarez and H. Kong, "Fusion of LiDAR and Camera by Scanning in LiDAR Imagery and Image-Guided Diffusion for Urban Road Detection," 2018 IEEE Intelligent Vehicles Symposium (IV), Changshu, 2018, pp. 579-584, DOI: 10.1109/IVS.2018.8500401.

[5] X. Bingqian, G. Yanfeng and C. Zhimin, "Building LiDAR point cloud denoising processing through sparse representation," 2015 IEEE International Geoscience and Remote Sensing Symposium (IGARSS), Milan, 2015, pp. 585-588, DOI: 10.1109/IGARSS.2015.7325831.

[6] Y. Zhang, X. Xiong, M. Zheng and X. Huang, "LiDAR Strip Adjustment Using Multifeatures Matched With Aerial Images," in IEEE Transactions on Geoscience and Remote Sensing, vol. 53, no. 2, pp. 976-987, Feb. 2015, DOI: 10.1109/TGRS.2014.2331234. 
[7] Eun Soo Nam, M. S. Oh, H. Y. Kim and Y. J. Chong, "Eye safe laser radar using a microchip laser, 2-dimensional InGaAs/InP photodiode arrays and the bi-axial optical lens system," 2008 Asia-Pacific Microwave Conference, Macau, 2008, pp. 1-4, DOI: 10.1109/APMC.2008.4957915.

[8] C. K. Williamson and R. J. DeYoung, "Reduction of PMT signal-induced noise in lidar receivers using an external electrode," Technical Digest. Summaries of papers presented at the Conference on Lasers and ElectroOptics. Postconference Edition. CLEO '99. Conference on Lasers and Electro-Optics (IEEE Cat. No.99CH37013), Baltimore, MD, USA, 1999, pp. 553-554. ID: 19980236770.

[9] G. Cozzi et al., "Development of a SiPM-based detection module for prompt gamma imaging in proton therapy," 2016 IEEE Nuclear Science Symposium, Medical Imaging Conference and Room-Temperature Semiconductor Detector Workshop (NSS/MIC/RTSD), Strasbourg, 2016, pp. 1-5, DOI: 10.1109/NSSMIC.2016.8069393.

[10] A. K. Krizsan, S. A. Kis, J. Gal, G. Hegyesi and L. Balkay, "Simulation studies with SiPM arrays and LYSO crystal matrix analyzing a new readout scheme," 2012 IEEE Nuclear Science Symposium and Medical Imaging Conference Record (NSS/MIC), Anaheim, CA, 2012, pp. 26792680, DOI: 10.1109/NSSMIC.2012.6551608.

[11] M. F. Santangelo et al., "SiPM as novel optical biosensor transduction and applications," 2014 Fotonica AEIT Italian Conference on Photonics Technologies, Naples, 2014, pp. 1-4, DOI: 10.1109/Fotonica.2014.6843944.

[12] P. Busca et al., "A SiPM-based detection module for SPECT/MRI systems," 2015 IEEE Nuclear Science Symposium and Medical Imaging Conference (NSS/MIC), San Diego, CA, 2015, pp. 1-3, DOI: 10.1109/NSSMIC.2015.7582257.

[13] D. Meier, S. Mikkelsen, J. Talebi, S. Azman, G. Mæhlum and B. E. Patt, "An ASIC for SiPM/MPPC readout," IEEE Nuclear Science Symposuim \& Medical Imaging Conference, Knoxville, TN, 2010, pp. 1653-1657, DOI: $10.1109 /$ NSSMIC.2010.5874056.

[14] A. K. Krizsan, S. A. Kis, J. Gal, G. Hegyesi and L. Balkay, "Simulation studies with SiPM arrays and LYSO crystal matrix analyzing a new readout scheme," 2012 IEEE Nuclear Science Symposium and Medical Imaging Conference Record (NSS/MIC), Anaheim, CA, 2012, pp. 26792680, DOI: DOI: 10.1109/NSSMIC.2012.6551608.

[15] A. L. Goertzen et al., "Design and Performance of a Resistor Multiplexing Readout Circuit for a SiPM Detector," in IEEE Transactions on Nuclear Science, vol. 60, no. 3, pp. 1541-1549, June 2013, DOI: 10.1109/TNS.2013.2251661.

[16] B. W. Baumbaugh et al., "Studies of SiPM and Scintillation Plates with waveshifter fiber and SiPM readout," 2009 IEEE Nuclear Science Symposium Conference Record (NSS/MIC), Orlando, FL, 2009, pp. 846849, DOI: 10.1109/NSSMIC.2009.5402424.

[17] Z. Al-Daher, L. P. Ivrissimtzis and A. Hammoudeh, "Electromagnetic Modeling of High-Frequency Links With High-Resolution Terrain Data," in IEEE Antennas and Wireless Propagation Letters, vol. 11, pp. 12691272, 2012, DOI: 10.1109/LAWP.2012.2226135.

[18] Q. Zhou and U. Neumann, "A streaming framework for seamless building reconstruction from large-scale aerial LiDAR data," 2009 IEEE Conference on Computer Vision and Pattern Recognition, Miami, FL, 2009, pp. 2759-2766, DOI: 10.1109/CVPR.2009.5206760.

[19] K. Sanjaya, F. Henning and K. R. Purba, "3D LIDAR City Model Application and Marketing Plan Development," 2017 International Conference on Soft Computing, Intelligent System and Information Technology (ICSIIT), Denpasar, 2017, pp. 238-242, DOI: 10.1109/ICSIIT.2017.50.

[20] Shu Weng, Gang Zhao and Bin He, "Rapid reconstruction of 3D building models from aerial images and LiDAR data," 2010 3rd International Conference on Advanced Computer Theory and Engineering(ICACTE), Chengdu, 2010, pp. V2-195-V2-198, DOI: 10.1109/ICACTE.2010.5579254.

[21] Garnett, R.; Adams, M.D. LiDAR -A Technology to Assist with Smart Cities and Climate Change Resilience: A Case Study in an Urban Metropolis. ISPRS Int. J. Geo-Inf. 2018, 7, 161, DOI: 10.3390/ijgi7050161

[22] S. Gargoum and K. El-Basyouny, "Automated extraction of road feature using LiDAR data: A review of LiDAR applications in transportation," 2017 4th International Conference on Transportation Information and Safety (ICTIS), Banff, AB, 2017, pp. 563-574, DOI: 10.1109/ICTIS.2017.8047822

[23] M. Tan, B. Wang, Z. Wu, J. Wang and G. Pan, "Weakly Supervised Metric Learning for Traffic Sign Recognition in a LIDAR-Equipped Vehicle," in IEEE Transactions on Intelligent Transportation Systems, vol. 17, no. 5, pp. 1415-1427, May 2016, DOI: 10.1109/TITS.2015.2506182.

[24] A. N. Catapang and M. Ramos, "Obstacle detection using a 2D LIDAR system for an Autonomous Vehicle," 2016 6th IEEE International Conference on Control System, Computing and Engineering (ICCSCE), Batu Ferringhi, 2016, pp. 441-445, DOI: 10.1109/ICCSCE.2016.7893614.

[25] A. Ghassemi, K. Sato, K., MPPC, Technical note, Hamamatsu Photonics K.K., Cat. No. KAPD9005E01 Mar. 2017.

[26] Introduction to SiPM, TECHNICAL NOTE, SensL Technologies Ltd, rev. 6.0, February 2017.

[27] M. d. M. Silva and L. B. Oliveira, "Regulated Common-Gate Transimpedance Amplifier Designed to Operate With a SiliconPhotoMultiplier at the Input," in IEEE Transactions on Circuits and Systems I: Regular Papers, vol. 61, no. 3, pp. 725-735, March 2014, DOI: 10.1109/TCSI.2013.2283992.

[28] J. Huizenga, S. Seifert, F. Schreuder, H.T. van Dam, P. Dendooven, H. Löhner, R. Vinke, D.R. Schaart, A fast preamplifier concept for SiPM based time-of-flight PET detectors, Nuclear Instruments and Methods in Physics Research Section A: Accelerators, Spectrometers, Detectors and Associated Equipment, Volume 695, 2012, Pages 379-384 ,DOI: http://dx.doi.org/10.1016/j.nima.2011.11.012.

[29] A. Katz, A. Eshkoli, A. Feningstein, C. Jakobson and Y. Nemirovsky, "Design considerations of CMOS Si photomultiplier for long range LIDAR," 2017 IEEE International Conference on Microwaves, Antennas, Communications and Electronic Systems (COMCAS), Tel-Aviv, 2017, pp. 1-4, DOI: 10.1109/COMCAS.2017.8244772.

[30] P. Dorosz, M. Baszczyk, W. Kucewicz and Ł. Mik, "Low-Power FrontEnd ASIC for Silicon Photomultiplier," in IEEE Transactions on Nuclear Science, vol. 65, no. 4, pp. 1070-1078, April 2018, DOI: 10.1109/TNS.2018.2816239.

[31] F. Ciciriello, F. Corsi, F. Licciulli, C. Marzocca and G. Matarrese, "Time performance of voltage-mode vs current-mode readouts for SiPM's," 2015 6th International Workshop on Advances in Sensors and Interfaces (IWASI), Gallipoli, 2015, pp. 249-253, DOI: 10.1109/IWASI.2015.7184979.

[32] G. Barile, A. Leoni, L. Pantoli, L. Safari and V. Stornelli, "A New VCII Based Low-Power Low-Voltage Front-end for Silicon Photomultipliers," $20183 r$ International Conference on Smart and Sustainable Technologies (SpliTech), Split, 2018, pp. 1-4, INSPEC Accession Number: 18074673.

[33] Pantoli L, Barile G, Leoni A, Muttillo M, Stornelli V. A Novel Electronic Interface for Micromachined Si-Based Photomultipliers. Micromachines (Basel). 2018;9(10):507, DOI: 10.3390/mi9100507.

[34] L. Safari, G. Barile, V. Stornelli and G. Ferri, "An Overview on the Second Generation Voltage Conveyor: Features, Design and Applications," in IEEE Transactions on Circuits and Systems II: Express Briefs, DOI: 10.1109/TCSII.2018.2868744.

[35] J. Čajka and K. Vrba, "The Voltage Conveyor May Have in Fact Found its Way into Circuit Theory", AEU-International Journal of Electronics and Communications, vol. 58, no. 4, pp. 244-248, 2004, DOI: 10.1078/1434-8411-54100239.

[36] A.S. Sedra, K.C. Smith, "A second-generation current conveyor and its application,” IEEE Trans. Circ. Th., vol. 17, no. 1, pp. 132-134, Jan 1970, DOI: $10.1109 /$ TCT.1970.1083067.

[37] G. Ferri, N.C. Guerrini, Low-voltage low-power CMOS current conveyors. Dordrecht: Kluwer, 2003, DOI: 10.1109/81.622987.

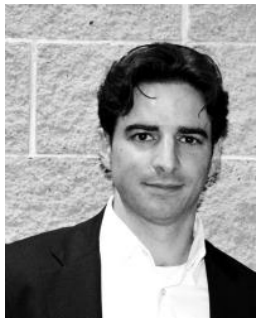

Leonardo Pantoli (M'09) received the Degree (cum laude) in Electronic Engineering and the Ph.D. in Electrical and Information Engineering from the University of L'Aquila, L'Aquila, Italy, in 2006 and 2010, respectively. In 2007 and 2008, he spent several months with the Communications Engineering Department of University of Cantabria, Spain and the C2S2 Department of the XLIM Research Institute, Brive La Gaillarde, France. He is Researcher with the University of L'Aquila and his research activities include the development of design methods for microwave nonlinear circuits, linear and nonlinear stability analysis, electronic interfaces for SiPMs and MMICs design for space and communication systems. 


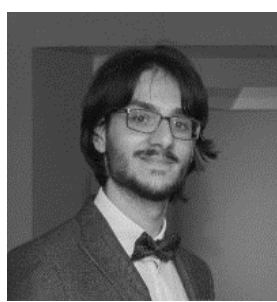

Gianluca Barile was born in Avezzano, Italy. He received the master's degree (cum laude) in Electronics Engineering from the University of L'Aquila (Italy) in 2016. He is currently involved as $\mathrm{PhD}$ Student at the University of L'Aquila. His research activity includes sensors interfaces, voltage and current mode integrated circuits and system for industrial electronics.

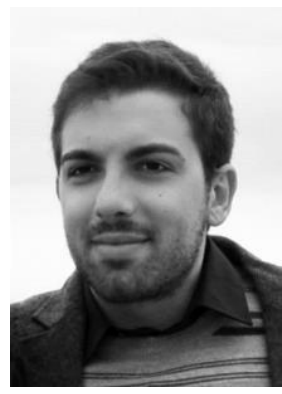

Alfiero Leoni was born in L'Aquila, Italy. He received the Bachelor "Laurea" degree in 2013 and master "Laurea" degree (cum laude) in electronic engineering in 2016. Several months before his master Laurea degree, he joined as an external collaborator the Department of Industrial and Electronic Engineering of the University of L'Aquila where he is enrolled as Ph.D. student, at the moment. His research activity mainly consists of the design of analog electronic and microelectronic circuits and systems for energy harvesting, industrial and microwave applications.

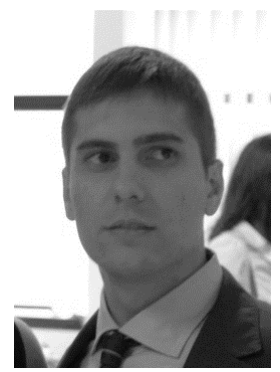

Mirco Muttillo was born in Campobasso, Italy. He received the master's degree (cum laude) in Electronic Engineering from University of L'Aquila, Italy, in 2016. He is a Ph.D. Student in Mechanical Engineering in the Department of Industrial and Information Engineering and Economics, University of L'Aquila. His research interests are concerning on design and analysis of electronic systems for energy efficiency and structural monitoring of buildings, problems in industrial electronics, and both analog and digital signal processing.

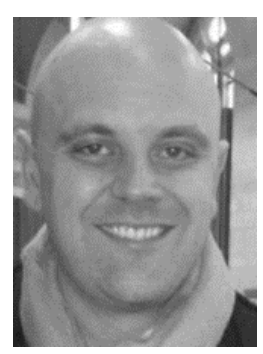

Vincenzo Stornelli was born in Avezzano, Italy. He received the "Laurea" degree (cum laude) in electronic engineering in 2004. In October 2004, he joined the Department of Electronic Engineering, University of L'Aquila, L'Aquila, Italy, where he is involved as Associate His research interests include several topics in computational electromagnetics, including microwave antenna analysis for outdoor ultrawideband applications. He serves as a reviewer for several international journals and Editor of the Journal of Circuits, Computers and Systems. 